\title{
Diffusion of $\beta$-lactam antibiotics into proteoliposomes reconstituted with outer membranes of isogenic imipenem-susceptible and -resistant strains of Enterobacter cloacae
}

\author{
Eun-Hee Lee, ${ }^{1}$ EkKehard Collatz, ${ }^{1}$ Joaquim Trias $^{2} \dagger$ and Laurent Gutmann ${ }^{1 *}$ \\ ${ }^{1}$ Laboratoire de Microbiologie Médicale, Université Paris VI, 15 rue de l'Ecole de Médecine, 75270 Paris Cedex 06, \\ France \\ ${ }^{2}$ Laboratoire de Bactériologie, CHU Pitié-Salpétrière, Université Paris-VI, 91 boulevard de l'Hôpital 75634 Paris \\ Cedex 13, France
}

(Received 8 May 1992; revised 6 July 1992; accepted 14 July 1992)

\begin{abstract}
The influence of outer membrane (OM) permeability on carbapenem susceptibility was studied in strains of Enterobacter cloacae, a species in which carbapenem resistance depends upon the conjunction of overproduction of the chromosomal cephalosporinase and reduction of $\mathrm{OM}$ permeability. Relative trans-OM diffusion rates were measured using the liposome swelling assay. Proteoliposomes were reconstituted with $\mathrm{OM}$ from the members of an isogenic set of $E$. cloacae strains, selected in vivo or in vitro, which produced either porins $F$ and $D$ (wild-type), or $F$ or D only, or neither. For all but one mutant, and compared with the wild-type strain, the respective increases in MICs and decreases in trans-OM diffusion of carbapenems were: nil and 13 to $18 \%$; 4 - to 32 -fold and 33 to $50 \%$; $\geq 64$-fold and $\geq 90 \%$. Our results suggest (i) that carbapenems (and other $\beta$-lactam antibiotics) diffuse through porins $F$ and $D$, but more rapidly through porin $F$, and (ii) that $O M$ permeability is the critical factor in determining the level of MICs of carbapenems for cephalosporinase-overproducing strains of $E$. cloacae. The OM of one particular low-level carbapenem-resistant and porin F- and D-deficient mutant was at least five times more permeable to carbapenems than the similarly porin-deficient high-level resistant mutants. We infer from this observation the possible existence of an alternative carbapenem penetration pathway which could be associated with two as yet uncharacterized overproduced OM proteins of about 22 and $47 \mathrm{kDa}$.
\end{abstract}

\section{Introduction}

Resistance to carbapenems can be acquired by mutations leading to the decreased production of specific or non-specific porins. In Pseudomonas aeruginosa, the reduction of one specific porin confers carbapenem resistance (Quinn et al., 1986; Trias et al., 1989; Trias \& Nikaido, 1991). In Enterobacter cloacae, resistance to carbapenems has also been described, in strains isolated in vivo (Lee et al., 1991) or selected in vitro (Lee et al., 1991; Raimondi et al., 1991). These strains produced very low amounts of non-specific porins and showed reduced penetration of carbapenems through the outer

* Author for correspondence. Tel. (1) 43292865 ; fax (1) 43256812

$\dagger$ Present address: Laboratori de Microbiologia, Facultat de Pharmacia, Universitat de Barcelona, 08028 Barcelona, Spain.

Abbreviations: OM, outer membrane(s); Cpm, carbapenem; Mox, moxalactam. membrane (OM). They simultaneously overproduced the chromosomal cephalosporinase that hydrolysed carbapenems at a very low rate. The conjunction of these two factors is thought to explain the carbapenem resistance observed in $E$. cloacae.

In this study, an isogenic set of $E$. cloacae strains, derived from $E$. cloacae 200 (Lee et al., 1991) and producing different amounts of porins, was analysed to better understand the influence of outer membrane permeability on carbapenem susceptibility. This was done by measuring the diffusion of carbapenems and other $\beta$-lactam antibiotics into proteoliposomes reconstituted with $\mathrm{OM}$ from the isogenic set of $E$. cloacae strains.

\section{Methods}

Bacterial strains and growth conditions. The E. cloacae strains used in this study are listed in Table 1. Strains 200, 201 and 201-Rev have been previously described (Lee et al., 1991). Strains 201-RevM1 and 201- 
Table 1. Enterobacter cloacae strains used in this study

\begin{tabular}{lll}
\hline \hline Strain & \multicolumn{1}{c}{ Relevant phenotype* } & \multicolumn{1}{c}{ Reference } \\
\hline 200 & $\mathrm{Cpm}^{\mathrm{S}} \mathrm{Mox}^{\mathrm{S}}$ clinical isolate & Lee et al. $(1991)$ \\
201 & $\mathrm{Cpm}^{\mathrm{R}} \mathrm{Mox}^{\mathrm{R}}$ mutant of strain 200 selected in vivo & Lee et al. $(1991)$ \\
201-Rev & $\mathrm{Cpm}^{\mathrm{S}} \mathrm{Mox}^{\mathrm{S}}$ spontaneous mutant of 201 & Lee et al. $(1991)$ \\
201-RevM1 & $\mathrm{Cpm}^{\mathrm{S}} \mathrm{Mox}^{\mathrm{R}}$ mutant of 201-Rev selected on moxalactam & This study \\
201-RevM3 & $\mathrm{Cpm}^{\mathrm{R}} \mathrm{Mox}^{\mathrm{R}}$ mutant of 201-Rev selected on moxalactam & This study \\
200-B3 & $\mathrm{Cpm}^{\mathrm{S}} \mathrm{Mox}^{\mathrm{R}}$ mutant of strain 200 selected on moxalactam & This study \\
\hline \hline
\end{tabular}

${ }^{*} \mathrm{Cpm}^{\mathrm{s}}$, carbapenem susceptible; $\mathrm{Cpm}^{\mathrm{R}}$, carbapenem resistant; $\mathrm{Mox}^{\mathrm{s}}$, moxalactam susceptible; Mox $^{\mathrm{R}}$, moxalactam resistant.

RevM3, derived from 201-Rev, and strain 200-B3, derived from $E$. cloacae 200 , were selected in vitro on moxalactam (16 to $\left.64 \mu \mathrm{g} \mathrm{ml}^{-1}\right)$.

All strains were grown at $37^{\circ} \mathrm{C}$ in Mueller-Hinton broth (Diagnostics Pasteur). The minimal inhibitory concentrations (MICs) of antibiotics were determined on Mueller-Hinton agar using a multiple inoculum replicator and $10^{4}$ c.f.u. per spot.

Antibiotics. The following antibiotics were kindly provided by the indicated companies: cefotaxime (Roussel-Uclaf, Paris, France); cefoxitin and imipenem (Merck Sharp and Dohme-Chibret, Paris, France); meropenem (ICI Pharmaceuticals, Alderley Park, UK); moxalactam (Eli Lilly and Sons, Indianapolis, Indiana, USA); cephaloridine (Glaxo, Paris, France); carbenicillin and ceftizoxime (SmithKline Beecham, Paris, France); ceftriaxone (Roche, Paris, France); cefacetril (Ciba-Geigy, Rueil-Malmaison, France).

Characterization of $O M$ proteins. All membranes were obtained from exponential-phase cultures $(200 \mathrm{ml})$ at an $\mathrm{OD}_{650}$ of 0.4 . Cells were harvested, washed in sodium phosphate buffer $(50 \mathrm{mM}, \mathrm{pH} 7.5)$ and broken by sonication in an ice bath for five to ten $30 \mathrm{~s}$ periods, separated by $30 \mathrm{~s}$ of cooling, with a Branson 3125 sonifier (Sonic Power Co.). OM were obtained in $0.3 \%(\mathrm{w} / \mathrm{v}) \mathrm{N}$-lauroylsarcosine (Sigma) as described previously (Gutmann et al., 1985). OM proteins were separated on polyacrylamide gels $(12 \%, \mathrm{w} / \mathrm{v})$ containing sodium dodecyl sulphate $(0.1 \%)$ and urea $(3 \mathrm{M})$. Proteins were visualized after silver staining (Heukeshoven \& Dernick, 1988).

Liposome swelling assay. Outer membranes were prepared as described above, except that they were additionally washed once in $5 \mathrm{mM}$-Tris/ $\mathrm{HCl}$ ( $\mathrm{pH} 7.5$ ) containing $0.3 \% N$-lauroylsarcosine and three times in $5 \mathrm{~mm}$-Tris $/ \mathrm{HCl}$ alone, and were resuspended in $400 \mu \mathrm{l}$ of the latter buffer. Liposomes were reconstituted according to the procedure described by Yoshimura \& Nikaido (1985) and by Nikaido \& Rosenberg (1983), except that $2.5 \mu \mathrm{mol}$ of acetone-extracted egg phosphatidylcholine, $0.1 \mu \mathrm{mol}$ of dicetylphosphate and 2-20 $\mu \mathrm{g}$ of OM proteins were used. The proteoliposomes were prepared in different solutions, depending upon the compounds to be tested in the swelling assay. (1) When the diffusion of non-charged compounds, including imipenem, which has a low solubility in $\mathrm{Tris} / \mathrm{HCl}$ buffer, was tested, a $5 \%(\mathrm{w} / \mathrm{v})$ Dextran T-40 solution was used (Trias et al., 1989). Approximately $20 \mu \mathrm{g}$ of protein were necessary under these conditions in order to measure the swelling rate of large compounds such as $\beta$ lactams. (2) When the diffusion of mono- and divalent anionic compounds was tested, a solution containing $1 \mathrm{~mm}$-imidazole/NAD, $4 \mathrm{~mm}-\mathrm{Na}_{2} \mathrm{NAD}$ and $12 \mathrm{~mm}$-stachyose was used (Nikaido \& Rosenberg, 1983).

The compounds to be tested were in $5 \mathrm{mM}$-Tris/ $\mathrm{HCl}$ buffer $(\mathrm{pH} 7.5)$ for sugars and zwitterionic compounds, and in $1 \mathrm{~mm}$-imidazole/NAD, $1 \mathrm{mM}-\mathrm{Na}_{2} \mathrm{NAD}(\mathrm{pH} \mathrm{6.0)}$ ) for mono- and divalent anionic compounds. The rates of diffusion through proteoliposomes were measured after
Table 2. MICs of $\beta$-lactam antibiotics for isogenic E. cloacae 200 derivatives

\begin{tabular}{|c|c|c|c|c|c|}
\hline \multirow[b]{2}{*}{ Strain } & \multicolumn{2}{|c|}{ Porin* } & \multicolumn{3}{|c|}{$\operatorname{MIC}\left(\mu \mathrm{g} \mathrm{ml}^{-1}\right)$} \\
\hline & $F$ & D & Imipenem & Meropenem & Moxalactam \\
\hline 200 & + & + & $0 \cdot 25$ & 0.06 & 4 \\
\hline 201 & - & - & 16 & 16 & 512 \\
\hline 201-Rev & + & - & 0.25 & 0.06 & 4 \\
\hline 200-B3 & - & + & 1 & 2 & 64 \\
\hline 201-RevM1 & - & - & 2 & 1 & 128 \\
\hline 201-RevM3 & - & - & 16 & 16 & 512 \\
\hline
\end{tabular}

*Porins in the different OM preparations: +, presence; -, absence.

dilution into isotonic solute solutions. All the results presented are the means of at least three determinations.

\section{Results}

\section{Antibiotic susceptibility and characterization of $O M$ proteins}

The MICs of imipenem, meropenem and moxalactam are shown in Table 2 and the profiles of the OM proteins of the different derivatives of $E$. cloacae 200 in Fig. 1 . The MICs of these compounds and the OM protein profiles were previously reported for strains 200, 201 and 201-Rev (Lee et al., 1991). For E. cloacae 201, 64- to 256fold increased MICs of carbapenems and moxalactam were correlated with the disappearance of two major OM proteins, with apparent molecular masses of 37 and 38 $\mathrm{kDa}$. Their acidic properties, revealed by two-dimensional gel electrophoresis (O'Farrell, 1975) (data not shown), are consistent with their being porins (Dang et al., 1988). Furthermore, the protein of $38 \mathrm{kDa}$ was suppressed after growth in high concentrations of glucose (data not shown). Thus, these two OM proteins should correspond to porins $\mathrm{F}$ and $\mathrm{D}$, respectively, as described by Raimondi et al. (1991). An additional 


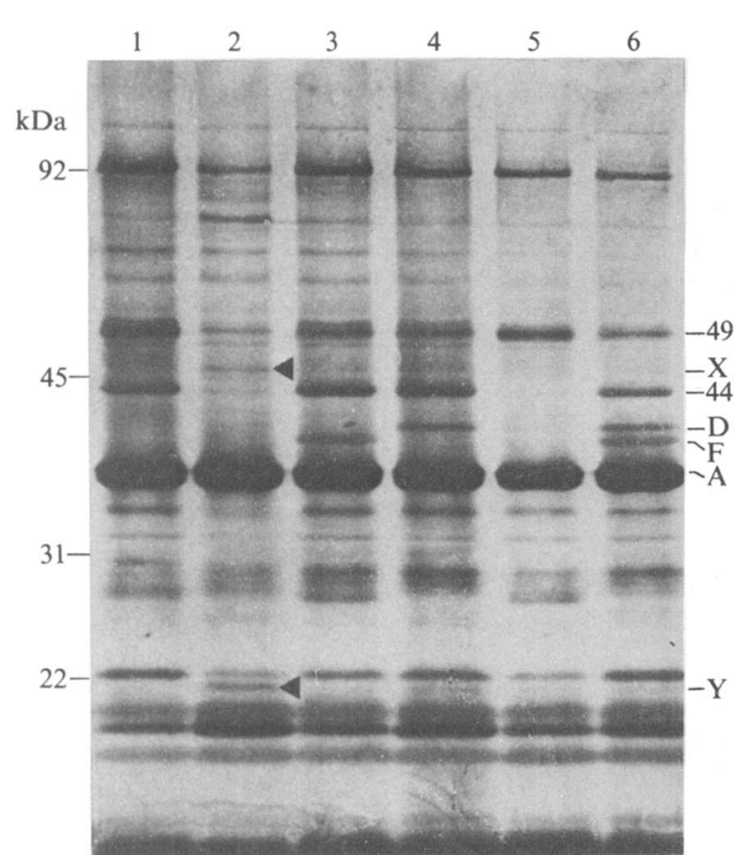

Fig. 1. SDS-PAGE of OM proteins of E. cloacae strains. Lanes : 1, 201RevM3; 2, 201-RevM1；3, 201-Rev; 4, 200-B3; 5, 201；6, 200. The positions of molecular mass markers are shown on the left. The positions of $\mathrm{OM}$ proteins are shown on the right: $\mathrm{X}$ and $\mathrm{Y}$, proteins typical of E. cloacae strain 200-RevM1; D, OmpD; F, OmpF; A, OmpA; 49 and $44 \mathrm{kDa}$ proteins of unknown function.

protein of about $44 \mathrm{kDa}$ was well visualized on the silverstained gel shown in Fig. 1 in strain 200 but not in strain 201. However, the relative amount of this protein varied greatly from one preparation to another. Under conditions revealing its presence in strain 200 it was always absent in strain 201. Its electrophoretic mobility, but not its relative amount, is reminiscent of that mentioned for porin C by Raimondi et al. (1991). In addition, and as shown previously (Lee et al., 1991), the quantity of a protein of about $49 \mathrm{kDa}$ increased somewhat in strain 201 (Fig. 1).

E. cloacae $201-\mathrm{Rev}$ regained $\beta$-lactam susceptibility, similar to that of $E$. cloacae 200 , in conjunction with the reappearance of porin F. E. cloacae 200-B3, which produced porin $\mathrm{D}$ and was deficient in porin $\mathrm{F}$, showed a 16-fold increased MIC of moxalactam and a 4- to 32-fold increased MIC of imipenem or meropenem (Table 2, Fig. 1). Strains 201-RevM1 and 201-RevM3 were derived from strain 201-Rev, which was deficient in porin $D$. They were representative of two populations selected on moxalactam at a frequency of between $10^{-8}$ and $10^{-7}$. Strain 201-RevM3 was deficient in porins $F$ and $\mathrm{D}$, just as the clinical isolate 201 , and showed an identical resistance phenotype. In contrast, strain 201RevM1, which was also deficient in porins $F$ and $D$,
Table 3. Swelling rates of proteoliposomes reconstituted with $O M$ from E. cloacae 200 and E. cloacae 201

Liposomes were made in $5 \%$ dextran.

\begin{tabular}{lccc}
\hline \hline \multirow{2}{*}{$\begin{array}{c}\text { Compound } \\
\text { tested }\end{array}$} & $\begin{array}{l}\text { Mol. } \\
\text { mass }\end{array}$ & \multicolumn{2}{c}{ Relative swelling rate* } \\
\cline { 3 - 4 } & $(\mathrm{Da})$ & E. cloacae 200 & E. cloacae 201 \\
\hline Arabinose & 150 & 100 & 15 \\
Glucose & 180 & 35 & 11 \\
Lactose & 360 & 4 & $<1$ \\
Imipenem & 299 & 15 & 1 \\
Cephaloridine & 415 & 6 & $<1$ \\
\hline \hline
\end{tabular}

* Results are expressed as percentages of the swelling rate of liposomes containing E. cloacae $200 \mathrm{OM}$, in the presence of arabinose.

Table 4. Swelling rates of proteoliposomes reconstituted with OM of E. cloacae 200 and E. cloacae 201

All the experiments were performed with liposomes containing the NAD imidazole buffer system.

\begin{tabular}{lllc}
\hline \hline \multirow{2}{*}{$\begin{array}{c}\text { Compound } \\
\text { tested }\end{array}$} & $\begin{array}{l}\text { Mol. } \\
\text { mass }\end{array}$ & \multicolumn{2}{c}{ Relative swelling rate* } \\
\cline { 4 - 4 }$(\mathrm{Da})$ & E. cloacae 200 & E. cloacae 201 \\
\hline $\begin{array}{l}\text { Zwitterionic compounds } \\
\quad \text { Cephaloridine }\end{array}$ & 415 & 100 & 6 \\
$\begin{array}{l}\text { Monoanionic compounds } \\
\quad \text { Cefacetril }\end{array}$ & 338 & 50 & 9 \\
Ceftizoxime & 382 & 39 & 11 \\
Cefoxitin & 426 & 41 & 5 \\
$\quad$ Cefotaxime & 454 & 24 & 2 \\
Dianionic compounds & & & \\
$\quad$ Carbenicillin & 376 & 11 & 2 \\
$\quad$ Moxalactam & 518 & 22 & 2 \\
Ceftriaxone & 552 & 20 & 5 \\
\hline \hline
\end{tabular}

* Results are expressed as percentages of the swelling rate of liposomes reconstituted with OM of E. cloacae 200, in the presence of cephaloridine.

showed only an 8- to 16 -fold increase in the MICs of carbapenems. Also, its OM protein profile differed from that observed for strains 201 and 201-RevM3 and showed overproduction of two proteins with apparent molecular masses of 22 and $47 \mathrm{kDa}$.

No differences in the composition of LPS of the different strains were observed (data not shown). The constitutive chromosomal cephalosporinase present in these strains (Lee et al., 1991) was produced at 5000 to $10000 \mathrm{mU} \mathrm{mg}^{-1}$ (using cephaloridine as substrate).

\section{Diffusion of $\beta$-lactam antibiotics}

In an attempt to correlate the different $O M$ protein patterns with the permeability of the OM and, consequently, with the susceptibility patterns of the various isogenic strains, their OM was incorporated into 
Table 5. Relative rates of diffusion of $\beta$-lactam antibiotics through proteoliposomes containing $O M$ of different derivatives of $E$. cloacae 200

Liposomes were reconstituted as described in Methods for the three zwitterionic compounds (imipenem, meropenem, cephaloridine) or the dianionic $\beta$-lactam moxalactam.

\begin{tabular}{|c|c|c|c|c|c|c|}
\hline \multirow{2}{*}{$\begin{array}{l}\text { E. cloacae } \\
\text { OM source }\end{array}$} & \multicolumn{2}{|c|}{ Porin* } & \multicolumn{4}{|c|}{ Relative swelling rate $\dagger$} \\
\hline & $\mathbf{F}$ & D & Imipenem & Meropenem & Cephaloridine & Moxalactam \\
\hline 200 & + & + & 100 & 100 & 100 & 100 \\
\hline 201 & - & - & 9 & 6 & 12 & 12 \\
\hline 201-Rev & + & - & 82 & 87 & 86 & 104 \\
\hline 200-B3 & - & + & 67 & 50 & 26 & 37 \\
\hline 201-RevM1 & - & - & 50 & 66 & 21 & 31 \\
\hline 201-RevM3 & - & - & $<5$ & $<5$ & $<5$ & 15 \\
\hline
\end{tabular}

*Porins in the different OM preparations: +, present; - , absent.

$\nmid$ Results are expressed as percentages of the swelling rate of liposomes reconstituted with OM proteins of E. cloacae 200.

proteoliposomes and the swelling rates in the presence of different sugars and antibiotic compounds were measured. Relative rates of diffusion into proteoliposomes reconstituted with OM prepared from the carbapenem-susceptible strain 200 and the resistant strain 201 (deficient in porins F and D) are shown in Table 3. The diffusion rate of arabinose into liposomes reconstituted with OM from the resistant strain 201 was $15 \%$ of that observed with liposomes reconstituted with $\mathrm{OM}$ from strain 200. Diffusion of the zwitterionic $\beta$-lactam imipenem through the OM of strain 201 was less than $10 \%$ of the diffusion observed through the OM of the susceptible strain 200 . When the OM of strain 200 was tested, diffusion of dianionic compounds was slower than that observed for monoanionic compounds, in particular carbenicillin (Table 4). All compounds tested showed a significantly reduced diffusion through the OM of the resistant strains.

When the permeability of the OM of E. cloacae 200derived strains was studied, a correlation between the diffusion of carbapenems (Table 5) and the MICs of these compounds (Table 2) was observed. Compared with strain 200, strain 201-Rev, which produced porin F, but not porin $\mathrm{D}$, and yielded no change in the MICs of these compounds, showed a relative transmembrane diffusion rate of $82 \%$. Strain $200-\mathrm{B} 3$, which produced porin $D$, but not porin $F$, and yielded a 4- to 32-fold increase in MICs of carbapenems, showed a greater decrease in transmembrane diffusion rate. For strains 201 and 201-RevM3, the deficiency in porins F and D and the at least 64-fold increase in the MICs of the carbapenems were associated with a diffusion rate through the OM which was less than $10 \%$ of that observed through the OM of the wild-type. In contrast, for strain 201-RevM1, which in spite of the apparent loss of the porins F and D showed only a 8- to 16 -fold increase in MICs of carbapenems, the diffusion rate of these compounds through its $\mathrm{OM}$ remained at 50 to $60 \%$ of that observed for the wild-type OM.

The lowest diffusion rate for moxalactam was observed through the OM of strains 201 and 201-RevM3 (deficient in porins $F$ and $D$ ), which showed the highest increase in MICs of this compound. It should also be pointed out that, when diffusion rates into proteoliposomes reconstituted with OM of strains 201-Rev and 200$\mathrm{B} 3$, producing only porin $\mathrm{F}$ or $\mathrm{D}$, respectively, were compared, lower diffusion rates were observed with the OM of strain 200-B3. This was associated with an increase in MICs of the different $\beta$-lactams for the latter strain, suggesting that the diffusion through an $\mathrm{OM}$ containing mainly porin $\mathrm{D}$, but not porin $\mathrm{F}$, is slower than the diffusion through an OM containing mainly porin $F$, but not porin $D$.

\section{Discussion}

The existence of three porins (F, D and C) has been reported for $E$. cloacae (Raimondi et al., 1991). Taking advantage of the selection of a set of derivatives of $E$. cloacae 200 which produced either no porins (E. cloacae 201 ), or porin $\mathrm{F}$ (E. cloacae 201-Rev), or porin D (E. cloacae 200-B3), we studied the contribution of the individual porins to the diffusion of different $\beta$-lactam antibiotics, and of carbapenems in particular. The apparent absence of porins $F$ and $D$ was associated with decreased diffusion of sugars and $\beta$-lactams. For strains 201 and 201-RevM3, deficient in porins $F$ and $D$, the increases in MICs of imipenem and meropenem (64- and 256 -fold) correlated with a decrease of at least $90 \%$ in the rate of diffusion of these compounds into proteoliposomes reconstituted with $\mathrm{OM}$. A similar decrease was observed in the diffusion of another zwitterionic compound, i.e. cephaloridine. This decrease in diffusion correlated with the eight-fold decreased permeability 
coefficient previously described for strain 201 using intact cells (Lee et al., 1991). The diffusion of anionic compounds, either mono- or dianionic, was also decreased in the same order of magnitude (see Table 4). Recovery of porin $F$ production in strain 201-Rev was sufficient for this mutant to regain a susceptibility to carbapenems equal to that of the wild-type strain 200. It was associated with an almost complete recovery of carbapenem diffusion through the OM. This would suggest that porin $\mathrm{F}$ alone allows enough diffusion of carbapenems to counteract the slow hydrolysis by the large amounts of cephalosporinase present in this strain, resulting in MICs similar to those observed in the F- and D-producing wild-type strain 200 . When porin D alone was produced, a 4- to 32-fold increase in MICs of carbapenems was associated with a more pronounced decrease in diffusion of larger $\beta$-lactams. These results taken together would suggest a difference in the poreforming properties of the two porins and a narrower pore diameter in porin $\mathbf{D}$.

Within the isogenic set of $E$. cloacae strains, 201RevM1 is very peculiar. This mutant, deficient in porins $\mathrm{F}$ and $\mathrm{D}$, was resistant to moxalactam but remained more susceptible to the carbapenems than strains 201 and 201-RevM3, which were also deficient in the two porins. When included into proteoliposomes, the OM of 201-RevM1 yielded reduced swelling rates compared to those obtained with the OM of the wild-type strain, but which were at least five-fold higher than those observed with the OM of the other F- and D-deficient strains. This would suggest that in this particular mutant an alternative diffusion pathway exists. Since no obvious changes in LPS and no quantitative changes in OmpA, which has been reported to have a pore-forming activity (Sugawara \& Nikaido, 1992), were obverved, we speculate that in strain 201-RevM1 (Fig. 1) one of the two overproduced OM proteins of apparent molecular masses 22 and 47 $\mathrm{kDa}$, or both, might allow diffusion of the carbapenems.

The production of at least the $47 \mathrm{kDa}$ protein is reminiscent of the overproduction of alternative porins in pseudorevertants generated from 'porinless' mutants of Escherichia coli (Bavoil \& Nikaido, 1977). Obviously, however, the possible pore-forming capacity of this protein needs to be tested experimentally.
This work was supported by grants from Merck Sharp and DohmeChibret and from the Institut National de la Santé et de la Recherche Médicale (CJF91-01).

We thank C. Harcour for secretarial assistance.

\section{References}

BavolL, P. \& NiKaIDO, H. (1977). Pleiotropic transport mutants of Escherichia coli lack porin, a major outer membrane protein. Molecular and General Genetics 158, 23-33.

Dang, P., Gutmann, L., Quentin, C., Williamson, R. \& Collatz, E. (1988). Some properties of Serratia marcescens, Salmonella parathyphi $A$ and Enterobacter cloacae with non-enzyme-dependent multiple resistance to $\beta$-lactam antibiotics, aminoglycosides, and quinolones. Reviews of Infectious Diseases 10, 899-904.

Gutmann, L., Williamson, R., Moreau, N., Kitzis, M. D., Collatz, E., ACAR, J. F. \& Goldstein, F. W. (1985). Cross-resistance to nalidixic acid, trimethoprim, and chloramphenicol associated with alterations in outer membrane proteins of Klebsiella, Enterobacter, and Serratia. Journal of Infectious Diseases 151, 501-507.

Heukeshoven, J. \& DeRNICK, R (1988). Improved silver staining procedure for fast staining in phastsystem development unit. I. Staining of sodium dodecyl sufate gels. Electrophoresis 9, 28-32.

Lee, E. H., Nicolas, M. H., Kitzis, M. D., Pialoux, G., Collatz, E. \& GutmanN, L. (1991). Association of two resistance mechanisms in a clinical isolate of Enterobacter cloacae with high-level resistance to imipenem. Antimicrobial Agents and Chemotherapy 35, 10931098.

NikAido, H., \& Rosenberg, E. Y. (1983). Porin channels in Escherichia coli: studies with liposomes reconstituted from purified proteins. Journal of Bacteriology 153, 241-252.

O'FarRell, P. H. (1975). High resolution two-dimensional electrophoresis of proteins. The Journal of Biological Chemistry 250, 4007-4022.

Quinn, J. P., Dudek, E. J., Divincenzo, C. A., Lucks, D. A. \& LERNER, S. A. (1986). Emergence of resistance to imipenem during therapy for Pseudomonas aeruginosa infections. Journal of Infectious Diseases 154, 289-294.

Raimondi, A., Traverso, A. \& Nikaido, H. (1991). Imipenem and meropenem resistant mutants of Enterobacter cloacae and Proteus rettgeri lack porins. Antimicrobial Agents and Chemotherapy 35, $1174-1180$

Sugawara, E. \& Nikaido, H. (1992). Pore-forming activity of Ompa protein of Escherichia coli. Journal of Biological Chemistry 267, 2507-2511.

Trias, J. \& NikaIDO, H. (1991). Outer membrane protein D2 catalyses facilitated diffusion of carbapenems and penems through the outer membrane of Pseudomonas aeruginosa. Antimicrobial Agents and Chemotherapy 34, 52-57.

Trias, J., Dufresne, J., Levesque, R. C. \& Nikaido, H. (1989). Decreased outer membrane permeability in imipenem resistant mutants of Pseudomonas aeruginosa. Antimicrobial Agents and Chemotherapy 33, 1201-1206.

YoshimURA, F. \& NIKaIDO, H. (1985). Diffusion of $\beta$-lactam antibiotics through the porin channels of Escherichia coli K12. Antimicrobial Agents and Chemotherapy 27, 84-92. 